\title{
OP IT MÊD
}

[1479]

J. Dijkstra, W. Heeringa, E. Yllmaz, $H$. van den Heuvel, D. van Leeuwen \& $H$. Van de Velde, 'A real time study of contact-induced language change in Frisian relative pronouns'. Yn: Elena Babatsouli (útj.), Proceedings of the International Symposium on Mono lingual and Bilingual Speech 2017, 113-119. http://www.ismbs.eu/publications-2017

Dit koarte stikje, dêr't likegoed mar leafst sân auteurs oan bydroegen ha, is in moaie yllustraasje fan de taalkundige mooglikheden fan it FAME-projekt, dat yn earste ynstânsje opset wie om it lûdargyf fan Omrop Frylân ûntslute te kinnen. Underwerp fan stúdzje is de saneamde bynwurdlike 't , dêr't Geart van der Meer yn 1991 alris fan úthâlden hat dat dy tebekrinne soe. Hjir binne allinnich de relative wurdsjes $d y$ 't, dêr't en wêr't ûndersocht, mar dy befêstigje yndied Van der Meer syn wiete finger. De /t/ waard yn it tiidrek 1966-1982 noch yn sa'n $85 \%$ fan 'e potinsjele gefallen útsprutsen, wylst dat persintaazje yn it tiidrek 2000-2015 sakke wie nei 60. It omslachpunt moat oars yn 'e tachtiger jierren west ha. Yn it earste tiidrek sjogge jo nammentlik dat de âldere generaasje wat leger útkomt as de jongere. Doe naam it gebrûk fan 'e bynwurdlike ' $t$ dus noch licht ta. De auteurs skowe

Us Wurk 68 (2019) s. 187-194; https://doi.org/10.21827/5d4811a271b50 dy opgong op in interne Fryske taalferoaring en de delgong op ynfloed fan it Nederlânsk, dat it ommers sûnder /t/ dwaan moat.

(siebren dyk)

Rolf H. Bremmer Jr, 'Latin loans in Old Frisian and the problem of relative chronology'. Yn: Claudia Di Sciacca, Concetta Giliberto, Carmela Rizzo \& Loredana Teresi (útjs.), Studies on Late Antique and Medieval Germanic Glossography and Lexicography in Honour of Patrizia Lendinara, vol. I. Pisa (2018), Edizioni ETA, 39-57.

Patrizia Lendinara is in Italiaanske germaniste mei ek in pear publikaasjes oer Aldfryske ûnderwerpen op har namme. Oan de feestbondel dy't har in skoftsje nei har ôfskie fan de universiteit fan $\mathrm{Pa}$ lermo oanbean is, hat Rolf Bremmer in artikel bydroegen oer Latynske lienwurden yn it Aldfrysk, yn it bysûnder de relative gronology dêrfan. Bremmer begjint mei fêst te stellen dat de stúdzje fan de Latynske lienwurden yn it Aldfrysk let op gong kommen is en dat der noch altiten gâns fragen binne. Dêrnei nimt er in tal foarbylden fan sokke lienwurden by de kop, dêr't er by sjen lit dat der oan dit type ûndersyk twa aspekten sitte, it iene lingwistysk, it oare kultureel. Yn it ûntlieningsproses ûnderskaat er trije perioaden: de Romeinske tiid, de tiid dy't 
kulminearret yn de kristening en fêstiging fan de tsjerke, en as lêste de dêrop folgjende perioade oant de ein fan de midsiuwen. Sa sydlings demonstrearret Bremmer ûnder oaren ek hoe't it Altfriesisches Handwörterbuch, hoe 'wonderful' oft it ek is, ûndersikers soms wol ris op 'e doele bringe kin, mei't by beskate lemma's nei de Aldfryske ek de Nederlânske en/of Leechdútske foarmen opjûn wurde, lykwols sûnder dat dy markeard wurde.

(oebele vries)

Rolf H. Bremmer Jr, 'Codifying the Law: Frisian Legal Manuscripts around 1300'. Yn: Erik Kwakkel (útj.), Vernacular Manuscript Culture 10001500. Studies in Medieval and Renaissance Book Culture. Leiden (2018), Leiden University Press, 143-185, 254259 (plate 11-17).

Yn dit beslist substansjele artikel docht Rolf Bremmer ferslach fan syn ferlykjend ûndersyk nei kodikologyske en paleografyske aspekten fan de âldste bewarre bleaune Aldfryske rjochtshânskriften en fragminten fan rjochtshânskriften, dy't - al as net tafallich allegearre ôfkomstich binne út Easterlauwersk Fryslân. Bremmer komt, op grûn fan yngeand en tige saakkundich útfierd ûndersyk, dat skerp offstekt by de gauris frijwat ympresjonistyske oanpak fan in eardere generaasje frisisten, ta inkelde wichtige konklúzjes. Yn it foarste plak kin it hânskrift R1 better datearre wurde, nammentlik op 'e tiid tusken 1250 en 1300 ynstee fan om 1300 hinne. Bremmer achtet it fierders sa goed as wis dat de hânskriften H1 en H2 fan de selde hân binne. Dêr komt dan noch by dat $\mathrm{H} 1$ earder ôfskreaun wêze moat as H2. De út de Ommelannen offkomstige pear 'flardsjes parkemint', sa't Jelle Brouwer dy yndertiid omskreau, datearret Bremmer op ' $\mathrm{e}$ tiid krekt foar 1400. Nijsgjirrich is syn observaasje dat de ûndersochte hânskriften, sa't bygelyks bliken docht oan de net al te bêste kwaliteit fan it parkemint dat brûkt is, wat 'ferskining' oanbelanget de sunige kant neist binne, wat goed past by de maatskiplike en bestjoerlike struktuer fan de doetiidske Fryske lannen.

(oebele vries)

Rolf H. Bremmer Jr, "'The fleeing foot is the confessing hand". Proverbs in the Old Frisian laws'. Yn: Marina Cometta, Elena Di Venosa, Andrea Meregalli \& Paola Spazzali (útjs.), La tradizione gnomica nelle letterature germaniche medievali. Milano (2018), Ledizioni, 79-100.

It ferneamdste Aldfryske sprekwurd is wol morth mot ma mith morthe kela, letterlik: moard moat men mei moard kuolje. Mei gâns mear Aldfryske foarbylden hat dat al yn de njoggentjinde iuw in plak krige yn Dútske sprekwurdsamlingen (Dútsk dan wol yn de breedste sin fan it wurd, d.w.s. mei ynbegryp fan Nederlânsk en ek Frysk). Eardere ûndersikers kwalifisearren dy Aldfryske sprekwurden as yn begjinsel oerâld ('urgermanisch'), dat der waard frijwat heech by opsjoen. Rolf Bremmer lykwols sjocht, ynspirearre troch benammen de ûntmytologisearjende observaasjes fan de germanist Klaus von See, mei in kritysk each nei al dat moais. Sa't Von See sjen liet dat yn it 
âldste Yslânske rjochtsboek, de Grágás, sprekwurden hielendal net foarkomme, mar krekt wol yn letmidsiuwske Skandinavyske rjochtsteksten, sa stelt ek Bremmer fêst dat de rykste boarne fan Aldfryske sprekwurden in letfyftjindeiuwsk rjochtsboek is, te witten Jurisprudentia Frisica. Dêryn fine wy sels in term foar it begryp 'sprekwurd', te witten byspilwird. Bremmer is oars wol ûnder de yndruk fan hoe't de ûnbekende gearstaller fan Jurisprudentia Frisica sprekwurden om him hinne struit dy't op leafst fjouwer boarnen weromgeane: Romeinsk rjocht, kanonyk rjocht, de bibel en dochs ek it âldere Fryske rjocht.

(oebele vries)

Jan Hallebeek, 'The Gloss to the Saunteen Kesta (Seventeen Statutes) of the Frisian Land Law', Tijdschrift voor Rechtsgeschiedenis 87 (2019), 30-64.

De om 1485 hinne printe ynkunabel fan it Frysje lânrjocht, yn 'e regel oantsjut as Druk, befettet net allinnich in lange rige Aldfryske rjochtsteksten, mar ek in moai protte Latynske glossen by dy teksten, 190 om krekt te wêzen. Oant no ta ha dy ferklearjende kanttekeningen mei ferwizingen nei it kanonike en Romeinske rjocht sa goed as gjin omtinken krige. Mei dit artikel fan de rjochtshistoarikus Jan Hallebeek is dêr no foargoed feroaring yn kommen, ek al giet it dêryn allinnich om de 29 glossen dy't by de tekst fan de Santjin Kêsten hearre. In wichtige konklúzje dy't Hallebeek lûkt, is dat de ûndersochte glossen net jonger wêze kinne as de twadde helte fan de fjirtjinde iuw. Fierders stelt de auteur fan dit artikel fêst dat de besoarger (of besoargers) fan de útjefte fan de ynkunabel, dy't, sa't al folle langer dúdlik is, de Aldfryske teksten frijwat behoffene hat, de glossen sa litten hat as se al wienen. Dat docht kleare bliken oan it feit dat de Aldfryske oanhalen dêr't dy glossen ornaris mei iepenje, kwa taalfoarm net oan dy fan de wól behoffene Aldfryske tekst oanpast binne. In oare konklúzje is dat de glossen der sa't it liket op rjochte binne om de ek yn de fjirtjinde iuw al net mear goed by de doetiidske juridyske realiteit passende Santjin Kêsten - dêr komme bygelyks noch asega's yn foar, dy't ûnderwilens al lang ferdwûn wienen - better brûkber te meitsjen foar de rjochtspraktyk fan de eigen tiid en dat sûnder dy iuwenâlde tekst sels te wizigjen.

(oebele vries)

Jan Hallebeek, Layci erant coiudices. Over de rol van leken in de Westerlauwerse seendprocedure. Rede in verkorte vorm uitgesproken bij het afscheid als hoogleraar Europese rechtsgeschiedenis aan de Vrije Universiteit te Amsterdam op 26 april 2019. Amsterdam (2019), Vrije Universiteit Amsterdam.

Ta de ferneamdste Aldfryske rjochtsteksten heart, njonken de Santjin Kêsten en de Fjouwerentwintich Lânrjochten, it Syndrjocht, oars sein it tsjerklik rjocht. Yn syn offskierede as heechlearaar rjochtskiednis giet Jan Hallebeek yn op ien beskaat aspekt fan dat rjocht, te witten de rol fan leken yn it tsjerklik gerjocht oftewol de synd. Dêrby sjocht er yn it bysûnder nei de Latynske glossen by it Syndrjocht sa't dy't yn de ynkunabel fan it Fryske lânrjocht te fi- 
nen binne. Yn twa idintike glossen wurdt beklamme dat de meirjochters yn it syndgerjocht leken wienen. De wurden dêr't dat mei bart (layci erant coiudices), fine wy werom yn de titel fan dizze ôfskierede. Hallebeek stelt fêst dat dit Syndrjocht yn alle gefallen sûnt de twadde helfte fan de fjirtjinde iuw plakmeitsje moatten hat foar eigen regelingen fan lokale syndgerjochten. De doe obsoleet wurden teksten lykje troch de glossen, dy’t Hallebeek rûchwei op 'e tiid tusken it midden fan de trettjinde en de ein fan de fjirtjinde iuw datearret, by de tiid brocht te wêzen, wat sa likernôch strykt mei wat er foar de glossen by de Santjin Kêsten fêststeld hat. It Syndrjocht sels liket him te stamjen út de earste helte fan de alfte iuw. Dêr sit er mei op ien line mei Van Buijtenen, dy't yn syn proefskrift út 1953 ta deselde konklúzje kommen wie. De rol fan leken as meirjochters yn it syndgerjocht is neffens Hallebeek net unyk, mar bysûnder is wol dat dy yn Fryslân sa lang bestean bleaun is.

(oebele vries)

Jan Hallebeek, 'Het Freiteil in laatmiddeleeuws Friesland'. Yn: G.P. van Niftrik, J. de Vries en M. de Wilde (red.), De Achterkant van Minerva. Opstellen aangeboden aan prof. Kees Cappon ter gelegenheid van zijn afscheid van de Universiteit van Amsterdam. Amsterdam (2019), Faculteit der Rechtsgeleerdheid, 13-24.

Under Freiteil wurdt ferstien it diel fan it fermogen dat in testator yn it belang fan syn sieleheil tamakket oan 'e tsjerke of oan goeddiedige ynstellingen. De fraach is hoe grut oft dat diel yn it letmidsiuwske Fryslân wie. Hallebeek freget yn dizze bydrage it omtinken foar in post yn it Aldfryske rjochtsboek Jurisprudentia Frisica (46:31), dêr't koart en krêftich yn gearfette wurdt wat it kanonike rjocht yn dy tiid oer it Freiteil learde. Fierders lûkt er de konklúzje dat it yn Jurisprudentia Frisica net sa lyk giet om it neilitten fan trije kear in treddepart, mar earder om it neilitten oan trije soarten fan bestimmingen.

(oebele vries)

Han Nijdam, 'The Body Legal in Frisian Law: Bridging the Gap Between the Lex Frisionum and the Old Frisian Compensation Tariffs'. Yn: Stefan Jurasinski \& Andrew Rabin (útjs.), Languages of the Law in Early Medieval England. Essays in Memory of Lisi Oliver. Leuven-Paris-Bristol (2019), Peeters, 101-126.

De kompensaasjetariven foar ferwûningen foarmje de kearn fan de Lex Frisionum (lette achtste iuw). Yn dit artikel stelt Han Nijdam de fraach oan 'e oarder oft der kontinuïteit bestiet tusken de tariven út dizze hiel betide Latynsktalige boarne en dy út de folle lettere Aldfryske rjochtsteksten, yn it bysûnder de iersten dêrfan. Hy begjint mei in neiere yntroduksje fan sawol de Lex Frisionum, dy't lestich is troch syn komplekse struktuer, as de ierste Aldfryske rjochtsteksten. Nijdam argumentearret dat der no genôch bewiis is dat it begjin fan de skriftlike optekening fan dy rjochtsteksten socht wurde moat yn de alfte iuw. Dat giet yn tsjin Rolf Bremmer syn betinken dat der foar 1200 yn de Fryske lannen noch gjin ynfrastruktuer bestie foar sa'n opteke- 
ning, mar slút dêrtsjinoer oan by de tradisjonele fisy. De ôfstân yn tiid tusken de beide ûndersochte tekstgroepen is dus neffens Nijdam minder grut as oft dy wêze soe as en Bremmer syn fisy folget. Dêrnei wiist de auteur in tal foarbylden fan kontinuïteit tusken de Lex Frisionum en de iere Aldfryske kompensaasjetariven oan en wol op twadderlei mêd: yn beskate oantsjuttingen fan ferwûningen en yn beskate taryfhichten. Der binne oars ek wol opfallende foarbylden op te neamen fan diskontinuïteit. Dat hoecht ús neffens Nijdam lykwols gjin nij te dwaan, want de teksten ha har no ienris hyltiten fierder ûntwikkele.

(oebele vries)

Jörg Peters, 'Saterland Frisian', Journal of the International Phonetic Association 49 (2019), 223-230.

It tydskrift fan de International Phonetic Association hat in searje "Illustrations of the IPA", dêr't de fonetyk fan aparte talen koart yn beskreaun wurde. De Oldenburger heechlearaar Jörg Pe- ters is blykber frege om it Sealter Frysk foar syn rekken te nimmen, wat resultearret yn in moai handich oersjoch. Nei in koarte yntroduksje fan taal en taalsitewaasje komme respektyflik de bylûden, lûden en twilûden op it aljemint. Der wurdt ek koart omtinken jûn oan klam en yntonaasje en it stik einiget mei twa transkripsjes (fonemysk en allofoanysk) fan in eintsje Sealter tekst. Dizze bydrage is basearre op eardere literatuer en op ûndersyk by ynforman- ten en hat ek each foar hjoeddeiske taalferskowings.

(siebren dyk)

Jelke Bloem, Arjen Versloot en Fred Weerman, 'Modeling a historical variety of a low-resource language: Language contact effects in the verbal cluster of Early-Modern Frisian'. Yn: Proceedings of the 1st International Workshop on Computational Approaches to Historical Language Change 2019 (pp. 265-271).

https://www.aclweb.org/anthology/W1 9-4733

It is bekend dat de "Hollânske" folchoarder by tiidwurdkloften (dat er hie sjoen ynstee fan (...) sjoen hie) yn earder iuwen ek al wol foarkaam. Op grûn fan de kwantiteit fan de foarkommende patroanen yn it ier-nijfryske korpus wurde hjir konklúzjes lutsen oangeande it aard fan meartaligens yn de doetiidse Fryske mienskip. Spitigernôch bart dat sa ymplisyt en koart om 'e hoeke dat ien en oar net goed benei te kommen is. Dit stik hie der by wûn as ien en oar folle better útlein wie, c.q. as der mear romte foar it eigentlike probleem ynromme wie. Alle útweidings oer taalsitewaasje, databestannen en technikaliteiten en oare ferplichte nûmers hie men dêr byneed graach foar ynlevere sjoen. In krityske redaksje hie fansels ek al holpen.

(siebren dyk)

Tanneke Schoonheim, 'Oudfries en Oudnederlands, twee verwante talen in 
één woordenboek', Trefwoord (2019), https://ivdnt.org/images/stories/ onderzoek_en_onderwijs/publicaties/ trefwoord/oudfries-oudnederlandsschoonheim.pdf

Yn dizze Nederlânsktalige werjefte fan in yn 'e simmer fan 2019 yn Ljouwert holden Ingelsktalige lêzing op 'e 10de International Conference on Historical Lexicography and Lexicology jout Schoonheim in oersjoch fan it Aldfrysk dat ferskûle sit yn it Oudnederlands Woordenboek. Dy Aldfryske wurden komme út runeynskripsjes, psalmglossen en benammen ek plaknammen. Neffens Schoonheim hie dat yn dat wurdboek wol wat mear eksplisyt makke wurde kind en se pleitet dan ek foar in "woordenboek van het Oudnederlands en het Oudfries tot het jaar 1200 ". Of, alternatyf, foar in hiel nij wurdboek fan it Aldfrysk. "Het is de moeite waard om eens serieus te gaan kijken of een dergelijk project inmiddels niet levensvatbaar kan zijn".

(siebren dyk)

Frits van der Kuip en Janneke Spoelstra, 'Lexicografie van het Fries: een beknopt overzicht', Trefwoord (2019), https://ivdnt.org/images/stories/ onderzoek_en_onderwijs/publicaties/ trefwoord/lexicografie-van-het-

Fries.pdf

Op 'en nij in omwurke tekst fan de Ljouwerter konferinsje oer histoaryske leksikografy. De titel is treflik, wy fine hjir yndied in oersjoch yn it koart fan de Fryske leksikografy, al is dy dan wol beheind ta it Westerlauwerske Frysk. Yn tsjinstelling ta eardere oersjoggen binne hjir ek moaie plaatsjes by te finen. Oan 'e oarder komme de taalsitewaasje, Nijfryske wurdboeken en leksikografy fan it Alden Midfrysk. De auteurs wize der op dat, yn tsjinstelling ta de oare Westgermaanske talen, it Frysk foar de âldere taalfazen noch leksikografyske basisfoarsjennings mist.

(siebren dyk)

Koen Zondag, "De staveringskwestje soarret noch". In analyze fan materiaal berêstend by Tresoar oangeande it feroarjen fan de Frysk stavering, likernôch fan 1945-1976. [Ljouwert] (2019), Tresoar [48 siden].

Dit sil ien fan 'e lêste putten west ha dy't de dit jier ferstoarne Koen Zondag noch ûnderhannen hân hat. Hy hat de op Tresoar bewarre argyfdoazen fan it eardere pedagogysk advysburo fan 'e Fryske Akademy by de kop hân dy't materiaal befetten oer de staveringsreboeljes fan nei de oarloch, oant sis mar 1980. De stikken hat er kreas oardere en koart beskreaun neffens doaze en jiertal. Handich is ek it alfabetyske oersjoch fan de dramatis personae efteryn. Lokkigernôch hat Zondag it net inkeld by in ynvintarisaasje litten. Dêroan foarôfgeand fine we in goed tsien siden mei in soarte fan histoaryske synteze. Dêrby is it flink wat jierren letter dochs ek wol wer ferbjusterjend om te sjen hoefolle emoasjes oft staveringsdiskusjes altyd wer oproppe. Of, sa't perfester Klaas Fokkema hjir oanhelle wurdt: "It is foar de measte minsken makliker om fan godstsjinst te feroarjen as fan stavering".

(siebren dyk) 


\section{F. Rainer-en-dy (útjs.), Competition in Inflection and Word-Formation. Sprin- ger Switzerland (2019).}

Yn dizze bondel oer it tema kompetysje steane sels twa bydragen mei in mear of minder frisistyske eftergrûn. Anne Merkuur, Jan Don, Eric Hoekstra en Arjen Versloot hawwe yn "Competition in Frisian Past Participles" (s. 195-222) de mulwurden mei in dûbelde markearring by de kop. It giet dan om swakke tiidwurden mei in stam op - $d$ of $-t$, dy't de sterke útgong -en "liene", en de sterke tiidwurden mei in yn "e stam ta $n$ redusearre oarspronklike sterke útgong -en, dy't noch in ekstra $-d$ nimme. Foarbylden binne de mulwurden praten (út praat) en fûnd (út fûn). Beide oanpassings ha har oarsprong yn 'e súdwesthoeke. It nuvere is dat it type praten him hast wol oer it hiele taalgebiet wreide hat, wylst it type fûnd fierhinne hingjen bleaun is yn it (súd)westen. Foar dat opmerklike feit komt it fjouwertal ûnder oanfiering fan Merkuur no mei in ferklearring dy't oanslút by ideeën fan Yang en dy't der op del komt dat der in relevant produktiviteitsferskil is tusken de swakke en sterke tiidwurden. De produktive swakke kinne har dêrom wól wreidzje.

Arjen P. Versloot en Eric Hoekstra geane djipper yn op it tema "Blocking as a function of the nature of linguistic representations: where psycholinguistics and morphology meet" (s. 145166). It is in heechst teoretysk ferhaal wurden. Blokkearring is ornaris de oantsjutting foar it ferskynsel dat ien term foar in beskaat konsept it ûntstean fan in konkurrearjende term opkeart. It klassike foarbyld is it Ingelske stealer, dat wol mooglik is, mar likegoed net bestiet fanwegen it foarkommen fan thief. Versloot en Hoekstra jouwe in wat oar byld fan it ferskynsel. Op grûn fan modelteoretyske oerwagings komme de auteurs der op út dat it net sa lyk blokkearjen is wat der spilet, mar mear in striid om it bestean fan twa konkurrearjende foarmen, dêr't de iene it dan op 'en doer wint, omdat er mear selektearre wurdt, en de oare eventueel marginaal noch wat fierder heukerje kin. It ferhaal is hiel bot basearre op frekwinsje. Dingen as semantyk en prosody wurde kwealik achtslein, wylst dy yn taal faak ek in rol spylje, bygelyks yn de konkurrinsje tusken de Fryske efterheaksels -heid en -ens, dy't hjir as wichtich foarbyld oanhelle wurdt.

(siebren dyk)

Veronique De Tier, Anne-Sophie Ghyselen \& Ton van de Wijngaard (útjs.), De wondere wereld van de streektaalgrenzen. Lezingen gehouden op de internationale streektaalconferentie in Amsterdam op 13 oktober 2017. Leiden (2019), Stichting Nederlandse Dialecten.

Fan in konferinsje oer dialektgrinzen en yn ferbân dêrmei de erkenning fan streektalen is in moai boekje makke. Ien bydrage giet oer "Het Bildts: een Fries-Nederlands grensgeval" (s. 2739). Eric Hoekstra, Paulus van Sluis en Hans Van de Velde behannelje yn it stikje wat skiednis, sosjology en taalkundige skaaimerken fan it Biltsk. Hja komme der op út dat it in mingtaal is, wat nijs dat gjin Frysk is en gjin Nederlânsk. Op grûn dêrfan is der in oanfraach foar erkenning útgien, mar dy 
hat it net helle. Mar fanwegen in aktive rûnte fan frijwilligers en organisaasjes binne de auteurs dochs net pessimistysk oer de takomst fan it Biltsk.

It Frysk wurdt yn dit boekje ek noch neamd yn Sjef Barbiers syn bydrage "Fluïde dialectgebieden" (s. 15-25) oer syntaktyske ferskynsels en yn Wilbert Heeringa en John Nerbonne harres oer
"Verstaanbaarheid en de erkenning van streektalen" (s. 49-63). Nettsjinsteande oerienkomsten blykt it Frysk der op 'e kaart fan Nederlân en België dochs wol út te rinnen, dat datoangeande hoege we oer erkenning yn diel III fan it Europeesk hânfêst ynearsten noch net yn te sitten.

(siebren dyk) 